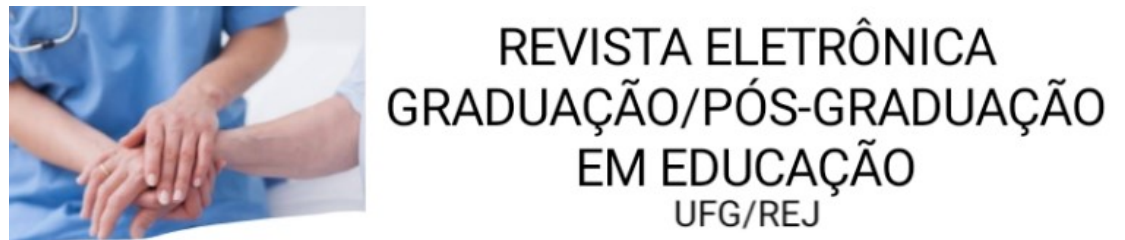

\title{
EdUCAÇÃo ESAÚdE-DosSIÊ DE ENFERMAGEM
}

\section{ÓBITOS POR CAUSAS MAL DEFINIDAS OCORRIDOS EM GOIÁS NO PERÍODO} DE 2010 A 2016

\author{
Cacia Regia de Paula ${ }^{1}$ \\ Ana Júlia Teodoro Rezende ${ }^{2}$ \\ Bruno Bordin Pelazza ${ }^{3}$ \\ Ludmila Grego Maia ${ }^{4}$ \\ Lucila Pessuti Ferri ${ }^{5}$ \\ Gleydson Alves Silva ${ }^{6}$ \\ Raul Henrique Oliveira Pinheiro 7 \\ Reila Campos Guimarães de Araújo ${ }^{8}$ \\ Juliana Flávia Ferreira e Silva Paranaíba ${ }^{9}$
}

RESUMO: Os indicadores epidemiológicos de mortalidade são imprescindíveis para o conhecimento do perfil da população. As estatísticas oficiais de mortalidade no Brasil são elaboradas a partir do processamento das informações presentes nas Declarações de Óbito. objetivo: descrever os óbitos por causas mal definidas no estado de Goiás, no período de 2010 a 2016. Materiais e métodos: trata-se de um estudo transversal e descritivo dos óbitos por CMD, utilizou-se o banco de dados do Sistema de Informação de Mortalidade (SIM) e DATASUS buscando pelas variáveis: sexo, faixa etária e local de ocorrência. Resultados: Do total de óbitos em Goiás no período de 2010 a 2016, 3,33\% foram de causas mal definidas. A maioria ficou concentrada na região metropolitana e sul-sudoeste do estado, e entorno do Distrito Federal. O sexo masculino e a faixa etária de 80 anos e mais, foram os de maior incidência. Foi possível evidenciar a redução dos óbitos por CMD no período analisado. Conclusão: os óbitos por CMD interferem diretamente em todos os indicadores de mortalidade, pois quanto menor a proporção, maior é a qualidade da informação no SIM. A avaliação periódica do banco de dados nos permite a identificação de falhas, asseguram

1 Enfermeira. Doutoranda em Enfermagem pelo Programa de Pós-Graduação de Enfermagem da Universidade Federal de Goiás (UFG). Professora do Curso de Enfermagem da Universidade Federal de Jataí (GO), Brasil. Email: caciaregia@gmail.com

${ }^{2}$ Enfermeira. Especialista em Saúde Pública. Assistente Técnica em Saúde da Secretaria da Saúde do Estado de Goiás - Regional de Saúde Sudoeste II - Jataí (GO), Brasil. Email: anajulia.rezende@bol.com.br

${ }^{3}$ Enfermeiro, Doutor em Ciências da Saúde pelo Programa de Pós-Graduação da Universidade Federal de Uberlândia (UFU). Professor do Curso de Enfermagem da Universidade Federal de Jataí (GO), Brasil. Email: bordizim@hotmail.com

${ }^{4}$ Enfermeira. Doutora em Ciências da Saúde pelo Programa de Pós-Graduação da Universidade Federal de Goiás (UFG). Professora do Curso de Enfermagem da Universidade Federal de Jataí (GO), Brasil. Email: Igregomaia@yahoo.com.br

5 Enfermeira. Mestre. Professora do Curso de Enfermagem da Universidade Federal de Jataí (GO), Brasil. Email: cilapessuti@bol.com.br

${ }^{6}$ Profissional de Educação Física. Especialista em Saúde Pública. Assistente Técnico em Saúde, Secretaria da Saúde do Estado de Goiás - Regional Sudoeste I. Rio Verde (GO), Brasil. Email: gleydsonalvesilva@gmail.com

Enfermeiro. Mestre pela Universidade de São Paulo- USP. Professor na Universidade Estadual do Centro Oeste do Paraná - UNICENTRO. Email: raulhop@icb.usp.br

8 Enfermeira, Doutoranda em Ciências da Saúde pelo Programa de Pós-Graduação da Universidade Federal de Goiás (UFG). Professora Mestre do Curso de Enfermagem da Universidade Federal de Jataí (GO), Brasil. Email: reilacampos@gmail.com

9 Bióloga, Especialista em Anatomia e patologia associada, Técnica Administrativa em Educação, Universidade Federal de Jataí, Curso de Medicina. (GO), Brasil. E-mail: julianaflaviaparanaiba@gmail.com 


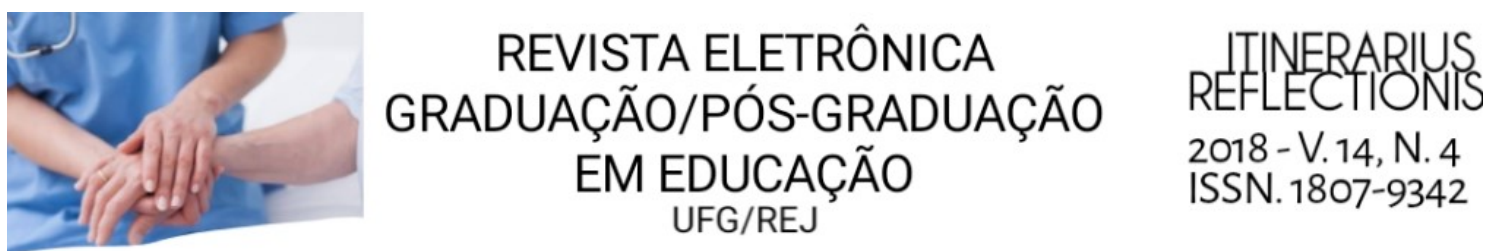

\section{EDUCAÇÃO ESAÚdE-DOSSIÊ DE ENFERMAGEM}

info

rmações de qualidade e possibilitam a implementação de políticas públicas a serem desenvolvidas.

Palavras-chave: Causas de Morte; Sistema de informação; Vigilância Epidemiológica.

\section{DEATHS FOR ILL-DEFINED CAUSES OCCURRED IN GOIÁS IN THE PERIOD OF 2010 TO 2016}

ABSTRACT: The Epidemiological indicators of mortality are indispensable for the knowledge of the population profile. The official mortality statistics in Brazil are elaborated from the processing of the information present in the Death Certificates.Objective: to describe the deaths from ill-defined causes in the state of Goiás, from 2010 to 2016. Materials and methods: this is a transversal and descriptive study of the deaths from ill-defined causes. It was used the database of the Mortality Information System (SIM) and DATASUS searching for variables: gender, age group and place of occurrence. Results: Of the total of the deaths in Goiás in the period from 2010 to $2016,3.33 \%$ were of ill-defined causes. The majority was concentrated in the metropolitan and south-southwest region of the state, and around the Federal District. Males and the age group of 80 years and over were the ones with the highest incidence. It was possible to evidence the reduction of deaths by CMD in the analyzed period. Conclusion: deaths due to CMD interfere directly in all mortality indicators, since the lower the proportion, the higher the quality of information in the Mortality Information System. The periodic evaluation of the database allows us to identify failures, ensure information of quality and enable the implementation of public policies to be developed.

Keywords: Causes of Death; Information System; Epidemiological surveillance. 


\section{REVISTA ELETRÔNICA GRADUAÇÃO/PÓS-GRADUAÇÃO EM EDUCAÇÃO UFG/REJ

\section{EduCAÇÃo ESAÚdE-DOSSIÊ DE ENFERMAGEM}

\section{INTRODUÇÃO}

Os indicadores epidemiológicos de mortalidade são imprescindíveis para o conhecimento do perfil da população. Estatísticas oficiais de mortalidade no Brasil são elaboradas a partir do processamento das informações presentes nas Declarações de Óbito (DO) (FRANÇA et al., 2014). A Organização Mundial de Saúde (OMS) em 1948 definiu o modelo internacional da DO que passou a ser usado no Brasil, e estabeleceu as regras de seleção para as Causas Básicas de Óbito (CBO) (MARTINS, BUCHALLA, 2015). A DO é uma ferramenta essencial para programar e avaliar as ações e investigações epidemiológicas. São fornecidas pelo Ministério da Saúde e distribuídas pelas secretarias estaduais e municipais de saúde aos estabelecimentos de saúde públicos e privados, regulamentada pela Portaria $\mathrm{n}^{\circ}$ 20 de 03/10/2003 (BRASIL, 2003).

De acordo com a OMS morte é definida como a cessação dos sinais vitais em qualquer momento após o nascimento com vida, sem possibilidade de ressuscitação. A verificação, constatação e declaração da morte do homem sob o ponto de vista biológico devem ser atestadas pelo médico, que tem a responsabilidade ética e jurídica pelo preenchimento da DO (LUCENA et al., 2014), sendo também o responsável pela especificação da CBO (JORGE et al, 2010).

Vilella et al., (2012) e Messias (2014) conceituam a CBO como: "doença ou lesão que iniciou a cadeia de acontecimentos patológicos que conduziram diretamente à morte, ou as circunstâncias do acidente ou violência que produziram a lesão fatal". Essas causas são tabuladas na Classificação Estatística Internacional de Doenças e Problemas Relacionados à Saúde - Décima Revisão (CID -10), possibilitando a comparação a nível nacional e internacional das análises estatísticas de morbimortalidade que impactam na saúde das populações.

O Sistema de Informação de Mortalidade (SIM) foi desenvolvido e informatizado pelo Ministério da Saúde (MS) em 1975, descentralizado para os estados e municípios em 1979, utiliza a CBO como fonte de dados de mortalidade, para o monitoramento do estado de saúde de populações e caracterização da situação sociodemográfica do país (SILVA et al., 2013). 


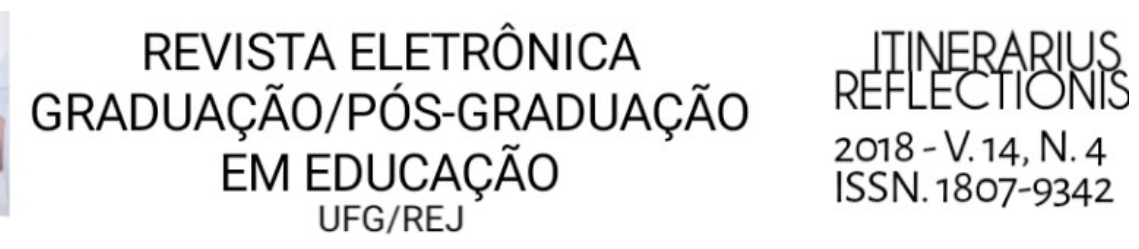

\section{EduCAÇÃo ESAÚdE-DossIÊ DE ENFERMAGEM}

A incompletude das informações na DO referente à causa básica (CB) faz com que esta seja classificada no capítulo XVIII da CID -10. Este capítulo aborda sintomas, sinais e achados anormais de exames clínicos e de laboratório não classificados em outra parte - Causas Mal Definidas (CMD), e de acordo com o Ministério da Saúde, este óbito deve ser investigado, sob o ponto de vista epidemiológico, para esclarecimento da causa básica utilizando como fontes os serviços de saúde de atenção primária, secundária e terciária, o Sistema de Informação de Agravos e Notificação (SINAN), Sistema de Informações Hospitalares, Serviço de Verificação de Óbito (SVO) e Instituto Médico Legal (IML), Boletins de Ocorrência Policial e entrevista no domicílio (BRASIL, 2011).

Considera-se a proporção de óbitos por CMD, um indicador de qualidade da informação sobre a causa básica do óbito, sendo que apresentando altas taxas, significa existência de dificuldade de acesso a serviços de saúde e de diagnóstico médico dos agravos e doenças que acometem a população, assim como dificuldade da qualidade e da assistência prestada pelos profissionais de saúde para elucidação do diagnóstico. Além disto, compromete a fidedignidade das estatísticas de mortalidade (FRANÇA et al., 2014 e RASELLA et al., 2014).

Uma importante ação para reduzir as CB de mortalidade, por CMD é a realização de análise das variações geográficas e temporais presentes nas DO, portanto as variáveis: sexo, faixa etária, causa básica, estado e ou município de ocorrência, são dados essenciais e os que melhor retratam o comportamento da mortalidade (LIU et al., 2015). Como o propósito de qualificar essas informações em 2010 o Ministério da Saúde procedeu com a reclassificação dos óbitos por CMD, utilizando-se da investigação epidemiológica, com isso neste mesmo ano, conseguiu-se a redução de 8,6\% para 7,0\% destes óbitos (FRANÇA et al., 2014).

O estado de Goiás, situado na Região Centro-Oeste, possui 257 municípios, dividido em 05 Macrorregiões de Saúde e subdividido em 18 Regionais de Saúde, com cobertura do Sistema de Informação de Mortalidade em todos os municípios. De acordo, com o SIM a proporção de óbitos por causas mal definidas no ano de 2015 em Goiás, por Macrorregião de Saúde, correspondiam a 2,28\% para região 


\section{REVISTA ELETRÔNICA GRADUAÇÃO/PÓS-GRADUAÇÃO EM EDUCAÇÃO UFG/REJ

\section{EDUCAÇÃo E SAÚdE-DOSSIÊ DE ENFERMAGEM}

Sudoeste, 2,17\% para região Sudeste, 2,39\% e 4,60\% para região Centro Norte e Nordeste respectivamente (SES-GO, 2017).

Diante disso, a escolha deste tema, se justifica pelo impacto da causa básica de óbito no perfil epidemiológico e no planejamento das políticas públicas. Por esta razão, este trabalho se propôs a descrever os óbitos por causas mal definidas ocorridos no Estado de Goiás, através do banco de dados do SIM, disponível no Departamento de Informática do Sistema Único de Saúde (DATASUS), no período de 2010 a 2016.

\section{MATERIAIS E MÉTODOS}

Trata-se de um estudo transversal, quantitativo, retrospectivo, dos registros de óbitos no SIM/DATASUS, dados de domínio público, conforme normatização da Resolução n 510, de 07 de abril de 2016 (BRASIL, 2016). Nesse sentido, não houve a necessidade de aprovação do Comitê de Ética em Pesquisa.

O presente estudo considerou todos os óbitos informados no SIM no período de 2010 a 2016, analisando os óbitos com causas básicas classificadas no Capítulo XVIII (Sintomas, Sinais e Achados Anormais de Exames Clínicos e de Laboratório não Classificados em Outra Parte), categoria de R00 a R99 da CID-10, com exclusão da categoria R95 e dos óbitos que não foram classificados no Capítulo XVIII. Os dados estatísticos foram usados pra descrever a distribuição das variáveis: faixa etária $(>1,1-4,5-9,10-14,15-19,20-29,30-39,40-49,50-59,60-69,70-79,80$ e mais e ignorado), sexo (masculino, feminino e ignorado), local de ocorrência do óbito (hospital, outro estabelecimento de saúde, domicílio, via pública, outros, ignorado) de residentes no Estado de Goiás.

Coletou-se os dados no sitio do DATASUS (http://www2.datasus.gov.br), sendo 2016 o último ano com dados disponíveis no sistema. Para o embasamento teórico, buscou-se ainda outros materiais eletrônicos, coletados no período de julho de 2016 a junho de 2018. 


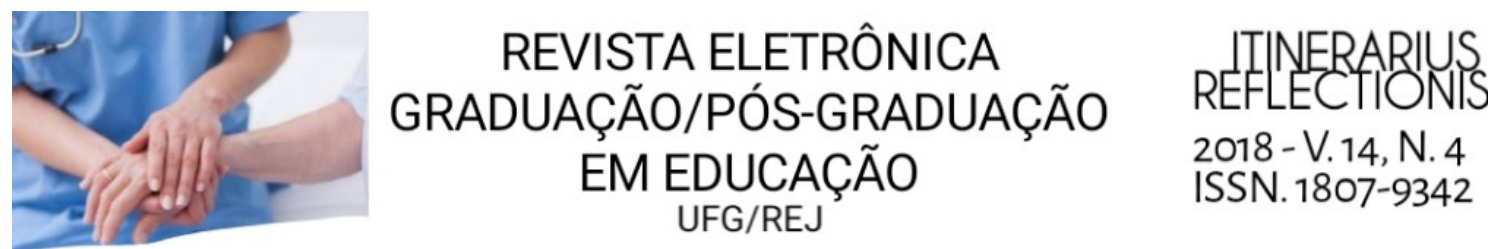

\section{EDUCAÇÃO E SAÚDE-DOSSIÊ DE ENFERMAGEM}

Os dados foram processados pelo programa Tabwin3, com programação dos arquivos de conversão, para representação em gráficos, figura e tabelas para análise dos dados sociodemográficos, local de ocorrência e categoria CID-10.

\section{RESULTADOS}

Observou-se que no estado de Goiás, de 2010 a 2016, ocorreram 253.800 óbitos, dos quais 3,33\% ( $n=8.452)$ foram classificados no Capítulo XVIII da CID -10 , na Categoria CID-10 de R00 a R99.

No tocante à distribuição espacial destes óbitos com causas mal definidas, é possível observar na figura 1 sua classificação dentre os 246 municípios do estado, havendo variação em forma de mosaico e aglomerados nas diversas regiões, cuja maior concentração está próxima ao Distrito Federal (DF) e na região Central do estado.

Figura 1. Óbitos com causas mal definidas segundo distribuição espacial no estado

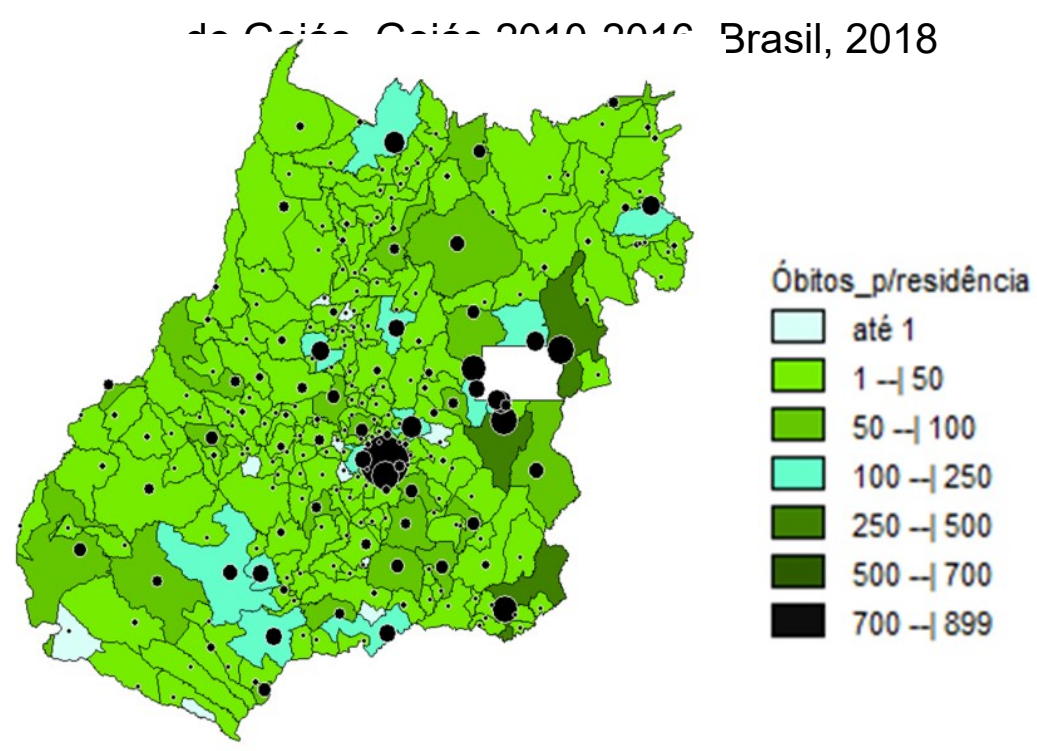

Fonte: MS/SVS/CGIAE - Sistema de Informações sobre Mortalidade - SIM, 2018.

Na tabela 1 observa-se a distribuição numérica e proporcional dos óbitos por CMD. Os dados demonstram que 53,30\% $(n=4.505)$ foram codificados por 


\section{REVISTA ELETRÔNICA GRADUAÇÃO/PÓS-GRADUAÇÃO EM EDUCAÇÃO UFG/REJ

\section{EduCAC̨Ão ESAÚdE-DossIÊ DE ENFERMAGEM}

tras Causas Mal Definidas e aquelas Não Especificadas, seguida pela Morte Sem Assistência Médica 15\% ( $\mathrm{n}=1.258)$.

Tabela 1. Óbitos com causas mal definidas segundo a Categoria CID-10. Goiás 2010-2016. Brasil, 2018

\begin{tabular}{lcc}
\hline \multicolumn{1}{c}{ Categoria CID - 10 } & $\mathrm{N}$ & $\%$ \\
\hline $\begin{array}{l}\text { R99 - Outras causas mal definidas e não } \\
\text { especificadas mortalidade }\end{array}$ & 4.505 & 53 \\
R98 - Morte sem assistência & 1.258 & 15 \\
R54 - Senilidade & 526 & 6 \\
R96 - Outras mortes súbitas de causa & 402 & 5 \\
desconhecida & 343 & 4 \\
R68 - Outros sintomas e sinais gerais & 150 & 2 \\
R57 - Choque não classificado em outra & 58 & 1 \\
parte & 59 & 1 \\
R64 - Caquexia & 1.051 & 12 \\
R10 - Dor abdominal e pélvica & 100 & 1 \\
R09 - Outros sintomas sinais relativo ao & 8.452 & 100 \\
\hline aparelho circulatório e respiratório & & \\
Outros & &
\end{tabular}

Fonte: MS/SVS/CGIAE - Sistema de Informações sobre Mortalidade - SIM, 2018.

A distribuição da frequência absoluta e relativa dos óbitos com causas básicas mal definidas foi realizada segundo sexo e faixa etária. O sexo masculino representa $63,41 \%(n=5.342)$ dos casos, sendo 80 anos e mais, a faixa etária mais atingida em ambos os sexos, correspondendo a $26,62 \%(n=2.243)$ do total (tabela 2). 


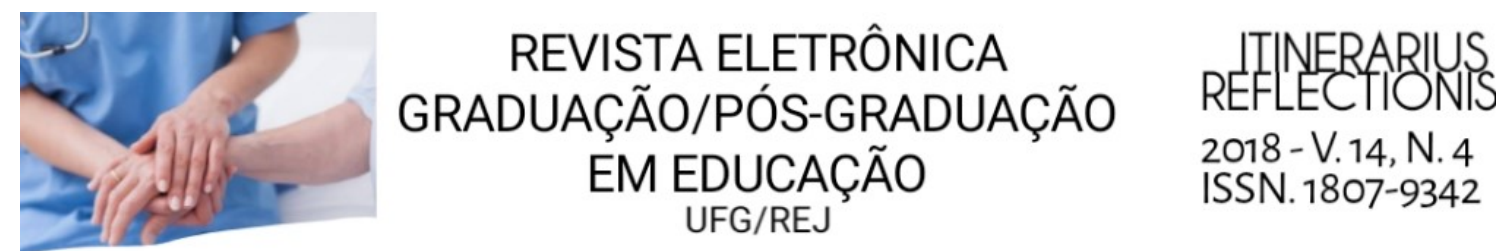

\section{EduCAÇÃo ESAÚdE-DossIÊ DE ENFERMAGEM}

Tabela 2. Óbitos com causas básicas mal definidas, segundo sexo e faixa etária.

Goiás 2010-2016. Brasil, 2018.

\begin{tabular}{ccccccc}
\hline $\begin{array}{c}\text { Faixa } \\
\text { etária }\end{array}$ & \multicolumn{2}{c}{ Masculino } & \multicolumn{2}{c}{ Feminino } & \multicolumn{2}{c}{ Ignorado } \\
(anos) & $\mathrm{N}$ & $\%$ & $\mathrm{~N}$ & $\%$ & $\mathrm{~N}$ & $\%$ \\
\hline$<01$ ANO & 83 & 1,5 & 67 & 2,2 & - & - \\
$01 / \mathrm{abr}$ & 29 & 0,5 & 22 & 0,7 & - & - \\
$05-09$ & 11 & 0,2 & 14 & 0,5 & - & - \\
$10-14$ & 24 & 0,5 & 12 & 0,4 & - & - \\
$15-19$ & 59 & 1,1 & 27 & 0,9 & - & - \\
$20-29$ & 235 & 4,4 & 92 & 3 & - & - \\
$30-39$ & 449 & 8,4 & 157 & 5,1 & 3 & 10,7 \\
$40-49$ & 579 & 10,8 & 245 & 7,9 & 1 & 3,6 \\
$50-59$ & 807 & 15,1 & 312 & 10,1 & - & - \\
$60-69$ & 922 & 17,3 & 370 & 12 & - & - \\
$70-79$ & 902 & 16,9 & 584 & 18,9 & - & - \\
80 e mais & 1.078 & 20,2 & 1.165 & 37,8 & - & - \\
Ignorado & 164 & 3,1 & 15 & 0,5 & 24 & 85,7 \\
Total & 5.342 & 100 & 3.082 & 100 & 28 & 100 \\
\hline
\end{tabular}

Fonte: MS/SVS/CGIAE - Sistema de Informações sobre Mortalidade - SIM, 2018.

Na figura 2 observa-se a distribuição dos óbitos com causas básicas mal definidas, segundo o local de ocorrência, com 51,54\% ( $n=4.356)$ no domicílio, seguido pela ocorrência em hospitais, $30,27 \%(n=2.558)$.

Figura 2. Óbitos com causas mal definidas segundo local de ocorrência. Goiás 20102016. Brasil, 2018.

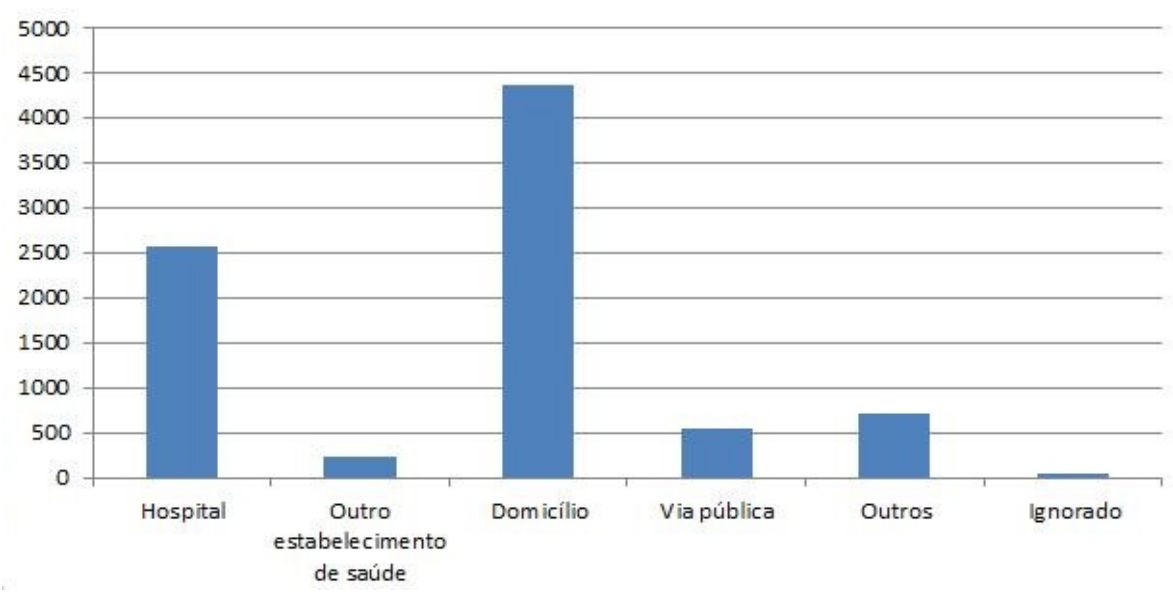




\section{REVISTA ELETRÔNICA GRADUAÇÃO/PÓS-GRADUAÇÃO EM EDUCAÇÃO UFG/REJ

\section{EDUCAÇÃo ESAÚdE-DOSSIÊ DE ENFERMAGEM}

Fonte: MS/SVS/CGIAE - Sistema de Informações sobre Mortalidade - SIM, 2018.

A Figura 3 apresenta a distribuição dos óbitos com causas básicas mal definidas, com redução progressiva de $23,40 \%$ ( $n=1.979)$ em 2010 , para $10,60 \%$ $(n=896)$ em 2016, resultando em uma redução de $12,80 \%$ no período analisado.

Figura 3. Óbitos com causas mal definidas segundo ano do óbito. Goiás 2010-2016. Brasil, 2018.

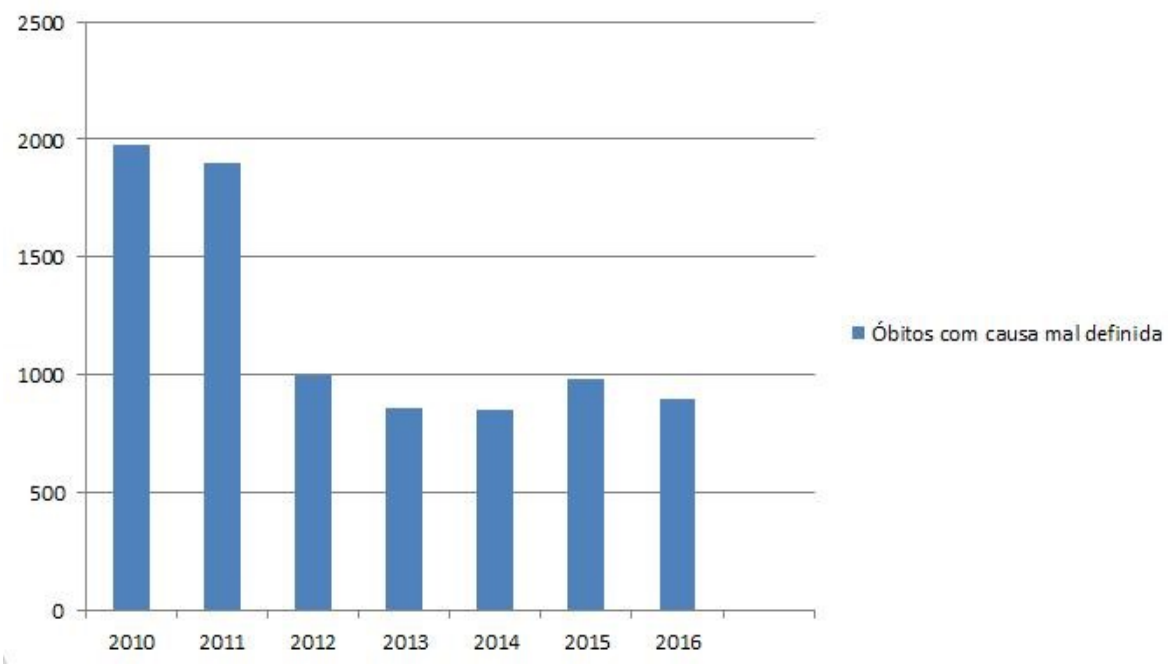

Fonte: MS/SVS/CGIAE - Sistema de Informações sobre Mortalidade - SIM, 2018.

\section{DISCUSSÃO}

A redução da proporção de óbitos com Causa Mal Definida é um desafio, com impacto direto na identificação da doença terminal e morte, nas mudanças no padrão de mortalidade e no perfil epidemiológico da população. Este estudo apresentou redução dos óbitos com CMD em Goiás, assim como observado nos países desenvolvidos e em desenvolvimento, com proporção de 12,80\%, considerada de boa qualidade segundo os parâmetros do Ministério da Saúde (BRASIL, 2014; MIKKELSEN et al., 2015). 


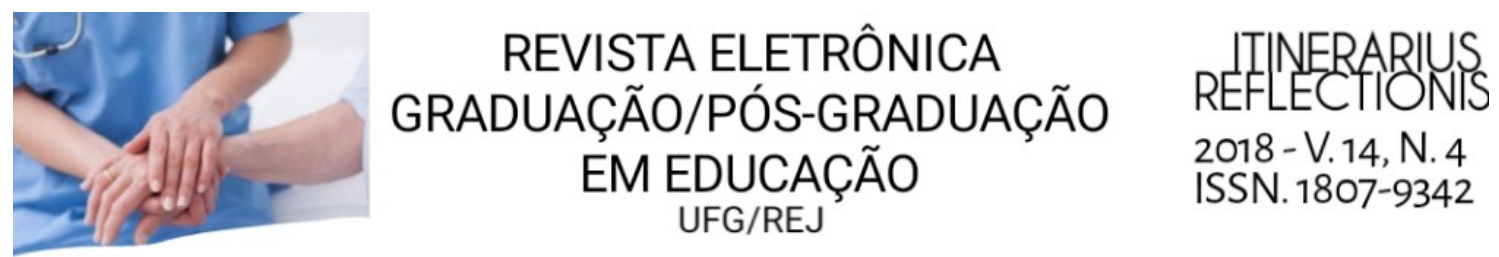

\section{EDUCAÇÃo ESAÚdE-DOSSIÊ DE ENFERMAGEM}

Ao comparar os óbitos por causas básicas mal definida informados no SIM/DATASUS, no total de 8.452 óbitos classificados no Capítulo XVIII da CID-10, as classificadas como Outras Causas Mal Definidas e as Não Especificadas representaram 53\%, seguida pela Morte Sem Assistência com 15\% e Outros sintomas e sinais relativos aos aparelhos circulatório e respiratório $12 \%$, consistindo em indicativo de problemas relacionados à qualidade dos dados da Declaração de Óbito (BRASIL, 2014; LUCENA et al., 2014; RASELLA et al., 2014; SILVA et al., 2015). A presença de CMD nas DO poderiam ser evitadas para muitos óbitos, caso os médicos buscassem informações complementares nos prontuários para esclarecer as causas de morte. Torna-se de extrema importância a conscientização do profissional médico sobre a relevância da DO, por meio de ações contínuas de capacitação que permitam o completo e correto preenchimento do documento, de forma que esses profissionais saibam especificar na $D O$ às condições e causas que levaram à morte (CUNHA; TEIXEIRA; FRANÇA, 2017).

Observa-se redução progressiva, de $12,80 \%$ no período de 2010 a 2016, e tendência estacionária, sugerindo melhora qualitativa das estatísticas de mortalidade nas variáveis analisadas. A proporção de óbitos com CMD entre estados, regiões e municípios pode apresentar variações. Neste trabalho foi possível observar que os óbitos com CMD apresentam 3, 33\% do total e quando analisados os municípios separadamente, analisa-se que a maior incidência está na região central do estado, avançando pela região metropolitana até próximo ao Distrito Federal. No ano de 2012 a proporção de óbitos por causa básica classificada no Capítulo XVIII da CID10 no Brasil foi de $6 \%$ e na Região Centro-Oeste de $3 \%$. Exemplo do que ocorre no Brasil, no estado de Goiás a incidência apresenta desigualdade entre municípios e macrorregiões (BRASIL, 2014; FRANÇA et al., 2014).

A análise dos dados destes óbitos quanto ao local de ocorrência, mostra que a maioria ocorreu no domicílio com $51,54 \%(n=4.356)$, seguido pela ocorrência em hospitais, 30,27\% ( $n=2.558$ ) (BRASIL, 2014), semelhante ao encontrado no estado do Piauí no período de 2000 a 2006, diferindo dos dados encontrados em Londrina no Paraná, que houve uma proporção inversa aos óbitos com CMD, que apresentou 


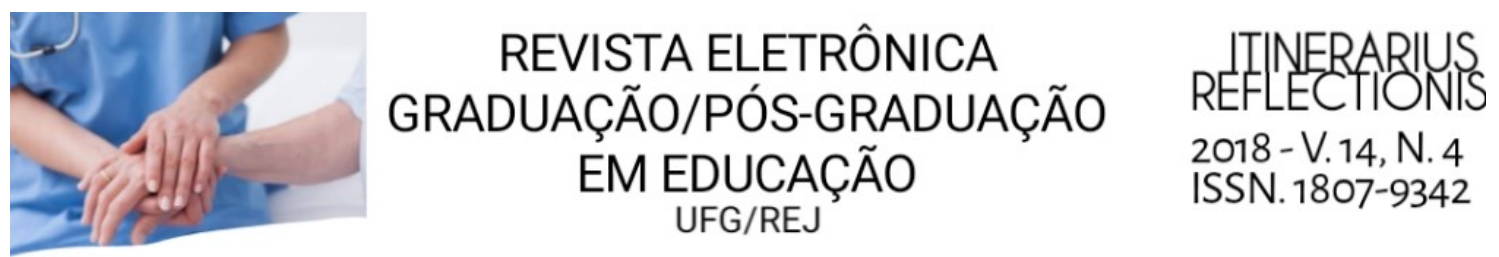

\section{EDUCAÇÃo E SAÚdE-DosSIÊ DE ENFERMAGEM}

com abordagem quantitativa e utilizando os métodos estatísticos para análise das variáveis, observaram-se melhora na qualidade da informação no Estado de Goiás, demonstrada pela redução na proporção dos óbitos com causas básicas mal definidas.

A predominância na faixa etária de idosos, maior ocorrência em domicilio, o alto percentual de outras CMD e não especificadas de mortalidade, a identificação dos municípios com alto percentual de óbitos com causas básicas mal definidas, refletem na caracterização do perfil epidemiológico e ressalta a necessidade de identificar os fatores desencadeantes e elaborar propostas de políticas públicas para que a proporção seja próxima de zero e de forma homogênea em todo o estado.

A avaliação periódica do banco de dados nos permite a identificação de falhas, asseguram informações de qualidade e possibilitam a implementação de políticas públicas a serem desenvolvidas.

É relevante ressaltar determinadas limitações deste estudo, como o fato de a pesquisa se limitar aos dados disponíveis no SIM e a confiabilidade dos mesmos relacionados ao preenchimento das DO`s, em virtude desta pesquisa ter como fonte dados secundários.

\section{REFERÊNCIAS}

BRASIL. MINISTÉRIO DA SAÚDE. PORTARIA No 20, DE 3 de outubro de 2003. Ministério da Saúde. Secretaria de Vigilância em Saúde. Publicada no DOU n¹96, de 9 de outubro de 2003, Secção I, p. 71. Disponível em:

<http://www.saude.sp.gov.br/resources/ccd/homepage/acesso-

rapido/civs/portaria_20_de_03_outubro.pdf> Acesso em: 13 de dezembro de 2017.

BRASIL. MINISTÉRIO DA SAÚDE. Secretaria de Vigilância em Saúde.

Departamento de Análise de Situação de Saúde. Manual de Instruções para o preenchimento da Declaração de Óbito / Ministério da Saúde, Secretaria de Vigilância em Saúde, Departamento de Análise de Situação de Saúde. - Brasília: Ministério da Saúde, 2011. 54 p. Disponível em:

<http://svs.aids.gov.br/download/manuais/Manual_Instr_Preench_DO_2011_jan.pdf> Acesso em: 06 de setembro de 2017. 


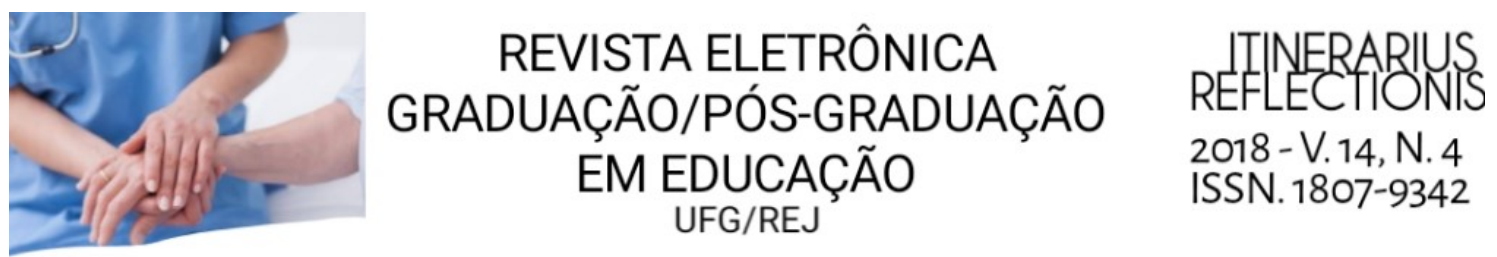

\section{EDUCAÇÃO ESAÚdE-DOSSIÊ DE ENFERMAGEM}

BRASIL. MINISTÉRIO DA SAÚDE. Secretaria de Vigilância em Saúde. Saúde Brasil 2013: uma análise da situação de saúde e das doenças transmissíveis relacionadas à pobreza: Ministério da saúde, 2014. Disponível em:

<http://bvsms.saude.gov.br/bvs/publicacoes/saude_brasil_2013_analise_situacao_sa ude.pdf > Acesso em: 30 de agosto de 2017.

BRASIL. MINISTÉRIO DA SAÚDE. Resolução $\mathbf{n}^{\circ}$ 510. Brasil, Ministério de Estado da Saúde. Publicada no DOU n 98, terça-feira, 24 de maio de 2016 - seção1, p. 4446. 2016. Disponível em:

<http://conselho.saude.gov.br/resoluções/2016/Reso510.pdf.> Acesso em: 30 de agosto de 2017.

BRASIL. MINISTÉRIO DA SAÚDE. Departamento de Informática do SUS. Datasus. Capítulo XVIII Sintomas, sinais e achados anormais de exames clínicos e de laboratório, não classificados em outra parte (R00-R99). 2017. Disponível em: <http://www.datasus.gov.br/cid10/V2008/WebHelp/r00_r99.htm> Acesso em: 29 de maio de 2018.

CUNHA, C.C.; TEIXEIRA, R.; FRANÇA, E. Avaliação da investigação de óbitos por causas mal definidas no Brasil em 2010. Epidemiol. Serv. Saude, Brasília, v.26, n.1, p.19-30, 2017.

FRANÇA, E.; TEIXEIRA, R.; ISHITANI, L.; DUNCAN, B. B.; CORTEZ-ESCALANTE, J. J.; NETO, O. L. M.; SCWARCWALD, C. Causas mal definidas de óbito no Brasil: método de redistribuição baseado na investigação do óbito. Revista Saúde Pública, v.48, n.4, p. 671-681, 2014. Disponível em:

<www.scielo.br/pdf/rsp/v48n4/pt_0034-8910-rsp-48-4-0671.pdf.> Acesso em: 10 de janeiro de 2018.

JORGE, M.H.P.M.; LAURENTI, R.; NUBILA, H.B.V.D. O óbito e sua investigação. Reflexões sobre alguns aspectos relevantes. Rev Bras Epidemiol, v.13, n.4, p. 561-76, 2010. Disponível em: <www.scielo.br/pdf/rbepid/v13n4/02.pdf>. Acesso em: 26 de outubro de 2018.

LAURENTI, R.; MELLO JORGE, M.H.P.; GOTLIEB, S.L.D. A confiabilidade dos dados de mortalidade e morbidade por doenças crônicas não-transmissíveis. Ciência \& Saúde Coletiva, v.9, n.4, p.909-920, 2004. Disponível em: <http://www.scielo.br/pdf/csc/v9n4/a12v9n4.pdf >. Acesso em: 25 de outubro de 2018.

LIMA, E.E.C.; QUEIROZ, B.L. Evolution of the deaths registry system in Brazil: associations with changes in the mortality profile, under-registration of death counts, and ill-defined causes of death. Cad. Saúde Pública, v.30, n. 8, p. 17211730, 2014. Disponível em: 


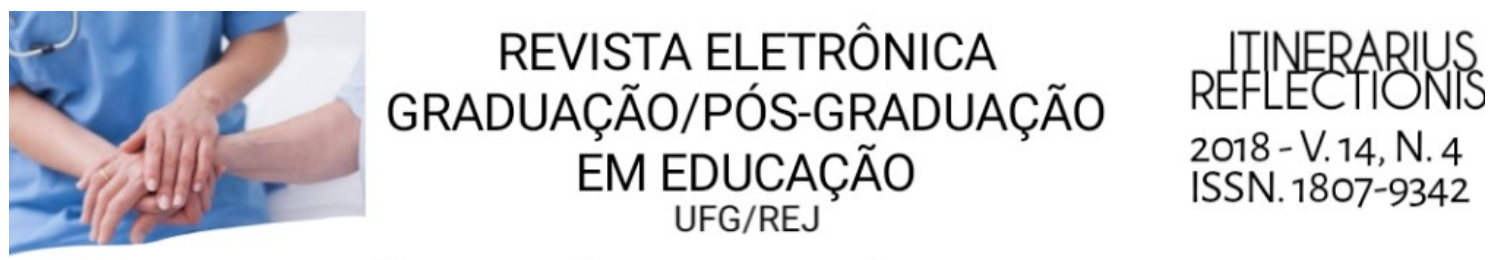

\section{EDUCAÇÃO ESAÚdE-DOSSIÊ DE ENFERMAGEM}

<http://www.scielo.br/scielo.php?script=sci_arttext\&pid=S0102311X2014000801721>. Acesso em: 10 de janeiro de 2017.

LIU, L; OZA, S; HOGAN, D. PERIN, J. RUDAN, I; LAWN, J; COUSENS, S.; MATHERS, C.; BLACK, R. Global, regional, and national causes of child mortality in 2000-13, with projections to inform post-2015 priorities: an updated systematic analysis. The Lancet, v.385, n.31, p. 430-40, 2015. Disponível em: <http://www.sciencedirect.com/science/article/pii/S0140673614616986>. Acesso em: 15 de fevereiro de 2018.

LUCENA, L; CAGLIARI, G.H.B; TANAKA, J; BONAMIGO, E.L. Declaração de óbito: preenchimento pelo corpo clínico de um hospital universitário. Revista Bioética, v. 22, n. 2, p. 318-324, 2014. Disponível em:

<http://www.scielo.br/pdf/bioet/v22n2/14.pdf> Acesso em: 29 de maio de 2018.

MARCUCCI, F.C.I.; CABRERA, M.A.S. Morte no hospital e no domicílio: influencias populacionais e das políticas de saúde em Londrina, Paraná, Brasil (1996 a 2010). Ciências e Saúde coletiva, v.20, n. 3, p. 833-840, 2015. Disponível em: <www.scielo.br/pdf/csc/v20n3/pt_1413-8123-csc-20-03-00833.pdf.>. Acesso em: 10 de janeiro de 2017.

MARTINS, R.C; BUCHALLA, C.M. Codificação e seleção automáticas das causas de morte: adaptação para o uso no Brasil do software Iris. Rev. Bras. de Epidemiol, v.18, n.4, p. 883-893. Disponível em: < http://www.scielo.br/pdf/rbepid/v18n4/19805497-rbepid-18-04-00883.pdf>. Acesso em: 26 de outubro de 2018.

MESSIAS, K.L.M. et al., Avaliação da qualidade das informações sobre óbitos por causas externas em Fortaleza, Ceará, Brasil. Ciência \& Saúde Coletiva, v.21, n.4, p.1255-1266, 2016. Disponível em: < http://www.scielo.br/pdf/csc/v21n4/14138123-csc-21-04-1255.pdf>. Acessado em 30 de outubro de 2018.

MIKKELSEN, L; PHILIPS, D.E.; ABOUZAHR, C; SETEL, P.W; SAVIGNY, D.; LOZANO, R.; LOPEZ, A.D. A global assessment of civil registration and vital statistics systems: monitoring data quality and progress. The Lancet, v.386, n.3-9, p.1395-406, 2015. Disponível em:

<https://www.thelancet.com/journals/lancet/article/PIIS0140-6736(15)601714/abstract?code=lancet-site> . Acesso em: 10 de janeiro de 2017.

OLIVEIRA, T.C; MEDEIROS, W.R; LIMA, K.C. Diferenças sociodemográficos da mortalidade de idosos em idades precoces e longevas. Rev. Baiana de Saúde Pública, v. 39, n. 2, p. 249-261, 2015. Disponível em:

<http://rbsp.sesab.ba.gov.br/index.php/rbsp/article/view/2063/pdf_622.>. Acesso em: 30 de agosto de 2017. 


\section{REVISTA ELETRÔNICA GRADUAÇÃO/PÓS-GRADUAÇÃO EM EDUCAÇÃO UFG/REJ

\section{EdUCAC̨ÃO ESAÚdE-DOSSIÊ DE ENFERMAGEM}

SELLA, D; HARHAY, M.; PAMPONET, M.L; AQUINO, R; BARRETO, M.L. Impact of primary health care on mortality from heart and cerebrovascular diseases in Brazil: a nationwide analysis of longitudinal data. BMJ, v. 349, p. 4014, 2014.

Disponível em: <http://www.bmj.com/content/349/bmj.g4014.short> Acesso em: 10 de janeiro de 2018.

SECRETARIA DE ESTADO DA SAÚDE DE GOIÁS- SES-GO. Mapa Interativo da Saúde do Estado de Goiás e do Brasil. Divisão Política e Regionalização.

Disponível em: <http://mapadasaude.saude.go.gov.br/mobile.php\#ficindic>. Acesso em 30 de agosto de 2017.

SILVA, J.A.C; YAMAKI, V.N; OLIVEIRA, J.P.S; TEIXEIRA, R.K.C; SANTOS, F.A.F; HOSOUME, V.S.N. Declaração de óbito, compromisso no preenchimento.

Avaliação em Belém - Pará, em 2010. Rev. Assoc. Med. Bras, v. 59, n. 4, p. 335340, 2013. Disponível em: <http://www.scielo.br/pdf/ramb/v59n4/v59n4a11.pdf> Acesso em: 15 de fevereiro de 2018.

VILLELA, L.C.M; REZENDE, E.M; DRUMOND, E.F; ISHITANI, L.H; CARVALHO, G.M.L. Use of the printed press for qualification of external causes of death. Rev. Saúde Pública, v. 46, n.4, p.730-736, 2012. Disponível em: http://www.scielo.br/scielo.php?pid=s003489102012000400018\&script=sci_arttext\&tIng=en>. Acesso em: 29 de maio de 2018. 